\title{
Regulating TLR-induced inflammation
}

Foster and colleagues report a new facet of regulation of the inflammatory response. They found that genes induced following Toll-like receptor 4 (TLR4) stimulation can be divided into two groups, the expression of which is differentially regulated as a result of TLR4-induced chromatin modifications.

Lipopolysaccharide (LPS) tolerance is a phenomenon of transient unresponsiveness that occurs following repeated LPS stimulation through TLR4. The authors speculated that it would be important for immune cells to maintain their ability to produce antimicrobial effector molecules even during a state of LPS tolerance in which tissue-damaging responses are limited, and as such, one would expect to see differential gene regulation of pro-inflammatory (tissue damaging) versus antimicrobial genes. Using an in vitro system of LPS tolerance in mouse macrophages, the authors tested this hypothesis and looked for differential gene regulation. Although the pro-inflammatory gene encoding interleukin- 6 was not inducible in LPS-tolerant macrophages, the antimicrobial gene encoding cathelicidin-related antimicrobial peptide was inducible.

Microarray analysis showed that several hundred genes induced during primary
LPS stimulation of macrophages were not inducible or were only inducible to a lesser degree upon secondary stimulation; these were therefore referred to as tolerizable genes. A second group of genes, referred to as non-tolerizable genes, were still inducible in LPS-tolerant macrophages. By analysing the kinetics of the expression of genes from the two different classes, performing chromatin immunoprecipitation assays and analysing the signalling pathways that contribute to gene induction, the authors showed that the two classes of genes are regulated by genespecific characteristics at the transcriptional level and not by differential signalling.

Next the authors looked at changes in histone modification and nucleosome remodelling, which are associated with gene-specific regulation. Histone acetylation is a feature associated with transcriptionally active chromatin, and the authors found that only genes in the non-tolerizable class were re-acetylated during LPS stimulation in tolerant macrophages. The histone modifications acetylation of histone 4 (H4) and trimethylation of $\mathrm{H} 3$ at lysine 4 (H3-K4) were observed in both types of genes in naive macrophages but not in tolerizable genes in tolerant macrophages. Using specific inhibitors of $\mathrm{H} 4$ acetylation and
H3-K4 trimethylation, the authors showed that inhibiting these changes turned tolerizable genes into non-tolerizable genes in tolerant macrophages. Furthermore, chromatin remodelling was inhibited at the promoters of tolerizable genes in tolerant macrophages, whereas non-tolerizable promoters were stable and inducibly remodelled.

Finally, the authors detected an element of adaptation in the innate immune response in that non-tolerizable secondary response genes - which are induced with later kinetics than primary response genes and the transcription of which is dependent on protein synthesis - are converted into primary response genes in tolerant macrophages as a result of initial LPS stimulation.

This study reveals that there are two groups of TLR4-induced genes that are differentially regulated at the level of chromatin modifications and it suggests that it might be possible to design an anti-inflammatory mediator for treating inflammation-mediated diseases while maintaining antimicrobial responses.

Elaine Bell

ORIGINAL RESEARCH PAPER Foster, S. L., Hargreaves, D. C. \& Medzhitov, R. Gene-specific control of inflammation by TLR-induced chromatin modifications. Nature 30 May 2007 (doi:10.1038/nature05836) 